\title{
Revisiting the Differential for Left Lower Quadrant Pain: A Case of Epiploic Appendagitis
}

\author{
Smith J L,2,4* and Potts $\mathrm{SE}^{3,4}$ \\ ${ }^{1}$ RNA Therapeutics Institute, University of Massachusetts \\ Medical School, Worcester, USA \\ ${ }^{2}$ Medical Scientist Training Program, University of \\ Massachusetts Medical School, Worcester, USA \\ ${ }^{3}$ Department of Family Medicine, University of \\ Massachusetts Medical School, Worcester, USA \\ ${ }^{4}$ Barre Family Health Center, UMassMemorial Health \\ Care, Barre, MA \\ *Correspondling author: J ordan L Smith, RNA \\ Therapeutics Institute, University of Massachusetts \\ Medical School; Medical Scientist Training Program, \\ University of Massachusetts Medical School; Department \\ of Family Medicine, University of Massachusetts Medical \\ School; Barre Family Health Center, UMassMemorial \\ Health Care, Barre, Worcester, MA 01605, USA
}

Received: December 30, 2020; Accepted: July 02, 2021; Published: J uly 09, 2021

\begin{abstract}
Appropriate triage of abdominal pain in the outpatient setting is critical for safe and effective management of patients. Abdominal pain has a broad differential, heavily dependent on elicitation of a clear history, and pertinent physical exam findings. In adults $>50$ with left lower quadrant pain, diverticulitis is the most common cause. Diverticulitis can cause significant morbidity in this population, frequently requiring inpatient management, systemic antibiotics, and occasionally surgical intervention. A frequently overlooked cause of left lower quadrant pain in adults is Epiploic Appendagitis (EA). While similar in presentation, unlike diverticulitis, EA rarely requires more than outpatient treatment with non-steroidal anti-inflammatories for pain management. Here we present a report of left lower quadrant pain consistent with diverticulitis in a 55 yo female in the outpatient setting, found to have EA by imaging. This case demonstrates that the differential for LLQ pain without signs of an acute abdomen in an adult, should include EA.
\end{abstract}

Keywords: Epiploic appendagitis; Diverticulitis; Acute abdomen; Triage; Outpatient

\section{Case Presentation}

A 55-year-old post-menopausal woman with a history of recurrent C. dificile infection, and urinary tract infections presented urgently to clinic with a complaint of acute left lower quadrant pain. She stated that the pain started approximately 48 hours ago. Her symptoms began with malaise, and she reports lying in bed all day. Around 24 hours prior to presentation, she had several episodes of loose watery stools; she describes them as "diarrhea-like." At presentation to the clinic, she has not had subsequent bowel movements in the last eighteen hours, but new onset crampy lower left abdominal. She endorses a sensation of bloating, and weight gain recently despite increased efforts in diet and exercise. She denies anorexia, nausea, fever, vomiting, dysuria, vaginal bleeding, hematochezia or melena. Her medical history is otherwise remarkable for chronic sciatic and lumbar radiculopathy historically managed with hydrocodone-acetaminophen 5-325 mg PO daily as needed, migraine with aura treated with butalbital with codeine 325-40 mg as needed, and gastroesophageal reflux managed with famotidine $20 \mathrm{mg}$ twice daily. Her surgical history was notable for a left partial oophorectomy. Of note, she had previous abdominal imaging in current year for unrelated symptoms with CT scan and MRI that did not reveal any obvious diverticula.

In the office, she was afebrile, normotensive at 124/81, and had a pulse of 91 . On exam, she is well appearing, well-nourished and not in acute distress, but she does have tenderness with palpation at the left lower quadrant to mid-flank. She does not have rebound or guarding. Her exam was otherwise normal. An in-office CBC 3-part auto differential was unremarkable. A clean-catch urine specimen was collected and found to be positive for $2^{+}$leukocyte esterase, trace blood, 70 WBCs (0-2 HPF), 6 RBCs (0-2 HPF), many bacteria and moderate mucus. Based on the history ascertained and in-office labs, a urinary tract infection was suspected with concomitant diverticulitis.
More remote possibilities for left lower quadrant pain with several episodes of loose stool included recurrent episode of C. dificile, nephrolithiasis, small bowel obstruction, appendicitis, mesenteric ischemia, and ischemic colitis. An outpatient CT Abdomen/ Pelvis with contrast and urinary culture were ordered. The patient was empirically started on ciprofloxacin $500 \mathrm{mg}$ BID for ten days to treat a presumed uncomplicated urinary tract infection, and possible uncomplicated diverticulitis.

The next day the CT Abdomen/Pelvis with contrast showed a $2.0 \mathrm{~cm}$ well circumscribed fat attenuation structure in the left lower quadrant near the descending sigmoid junction with adjacent mild soft tissue stranding and local peritoneal inflammation, consistent with Epiploic Appendagitis (EA). Urinary cultures showed growth of mixed flora, and suspected contamination. The patient had six total days of severe pain following diagnosis of epiploic appendagitis, and supportive care was administered including non-steroidal antiinflammatories for pain management.

\section{Discussion}

Recognition and appropriate triage of an acute abdomen is a critical skill in both outpatient and emergent care settings. Left Lower Quadrant (LLQ) pain is a frequent complaint, with diverticulitis representing the majority of cases in adults in the United States [1]. As such, the differential for acute and subacute left lower quadrant pain often begins with diverticulitis. As discussed by the American Academy of Family Physicians (AAFP), other frequent causes of left lower quadrant pain in a middle-aged female include other gastrointestinal causes like constipation, appendicitis, incarcerated hernias, infectious colitis, ischemic bowel, renal causes like ureterolithiasis and urinary tract infection, and gynecologic causes like endometriosis, malignancy, ruptured ovarian cyst, ovarian torsion, and rarely abdominal wall defects including hematoma, and 
abscess [2].

Often left off differentials for left lower quadrant, EA, unlike other causes of acute abdomen with similar presentation (i.e. diverticulitis, appendicitis) is typically self-limiting and does not routinely require surgical intervention or antibiotic use [3]. Inflammation, incarceration, infarction or ischemia of epiploic appendages, collectively known as EA, is a rare cause of left lower quadrant pain with the incidence estimated at 8.8 cases per million per year [4]. Epiploic appendages are fat-filled outpouchings of various sizes on the colon that contain a draining vein and arterial branch. The appendages occur in the greatest number in the sigmoid and transverse colon wall, and their function remains largely unappreciated [5,6]. Epiploic appendages are thought to be prone to infarction and ischemia because of their mobility, stalk-like shape, and limited arterial and venous vascular supply [7].

To date, there are no pathognomonic findings, or laboratory abnormalities associated with EA. Further, some clinical characteristics like obesity and older age associate with increased frequency of both diverticulitis and EA. Interestingly, EA has a slight predominance in males, while diverticulitis in adults over 50 is more common in women. Further, unlike diverticulitis which associates with a sedentary lifestyle, EA is more often observed in individuals who recently began a new exercise regimen [4,7]. A retrospective case control study of patients with EA versus diverticulitis, found that patients with diverticulitis more often presented with a more severe clinical presentation including fever, elevated white count, elevated inflammatory markers, nausea, and vomiting [7].

The most common presentation for EA is an afebrile middleaged patient with acute onset left lower quadrant pain without nausea and vomiting. The tenderness is not generalized and is frequently accompanied by rebound tenderness. Further, a mass can be palpated in the left lower quadrant of approximately $30 \%$ of patients [7]. EA is a radiographic diagnosis and requires abdominal CT for confirmation $[3,8,9]$. On CT, patients with EA often have a fatty lesion with a hyperattenuating ring, and bowel wall thickening $[8,10]$. EA is typically self-limiting and does not require the regular use of antibiotics, surgical intervention or hospitalization [9].

\section{Conclusion}

When a middle age patient presents with left lower quadrant pain with or without rebound guarding, EA should be included in the differential. EA frequently mimics diverticulitis, and appendicitis; however, the management of EA does not include a surgical consult, or antibiotics. The recognition of EA by radiologists frequently reduces the burden of unnecessary intervention; however, an awareness of EA's typical findings on CT imaging will further help to reduce unwarranted antibiotic use, and possible hospitalization for suspected diverticulitis. Clinicians should monitor patients with EA as outpatients and use NSAIDs for management of associated pain. In the event of recurrent EA or worsening symptoms, a surgical consult should be considered.

\section{References}

1. Ferzoco LB, Raptopoulos V, Silen W. Acute Diverticulitis. New England Journal of Medicine. 1998; 338: 1521-1526.

2. Hammond NA. Nikolaidis $P$, Miller FH. Left lower-quadrant pain: guidelines from the American College of Radiology appropriateness criteria. Am Fam Physician. 2010; 82: 766-770.

3. Sand M, Gelos M, Bechara FG, Sand D, Wiese TH, Steinstraesser L, et al. Epiploic appendagitis-clinical characteristics of an uncommon surgical diagnosis. BMC Surgery. 2007; 7: 11

4. de Brito P, Gomez MA, Besson M, Scotto B, Huten N, Alison D. [Frequency and epidemiology of primary epiploic appendagitis on $\mathrm{CT}$ in adults with abdominal pain]. J Radiol. 2008; 89: 235-243.

5. Breda Vriesman AC, Lohle PNM, Coerkamp EG, Puylaert JBCM. Infarction of omentum and epiploic appendage: Diagnosis, epidemiology and natural history. European Radiology. 1999; 9: 1886-1892.

6. Carmichael $\mathrm{DH}$, Organ, $\mathrm{CH}$. Epiploic Disorders: Conditions of the Epiploic Appendages. Archives of Surgery. 1985; 120: 1167-1172.

7. Hwang JA, Kim SM, Song HJ, Lee YM, Moon KM, Moon CG, et al. Differential diagnosis of left-sided abdominal pain: primary epiploic appendagitis vs colonic diverticulitis. World J Gastroenterol. 2013; 19: 6842-6848.

8. Almeida AT, Melão L, Viamonte B, Cunha R, Pereira JM. Epiploic Appendagitis: An Entity Frequently Unknown to Clinicians-Diagnostic Imaging, Pitfalls, and Look-Alikes. American Journal of Roentgenology. 2009; 193: 1243-1251

9. Schnedl WJ, Krause R, Tafeit E, Tillich M, Lipp RW, Wallner-Liebmann SJ. Insights into epiploic appendagitis. Nature Reviews Gastroenterology \& Hepatology. 2011; 8: 45-49

10. Singh AK, Gervais DA, Hahn PF, Sagar P, Mueller PR, Novelline RA. Acute Epiploic Appendagitis and Its Mimics. RadioGraphics. 2005; 25: 1521-1534. 